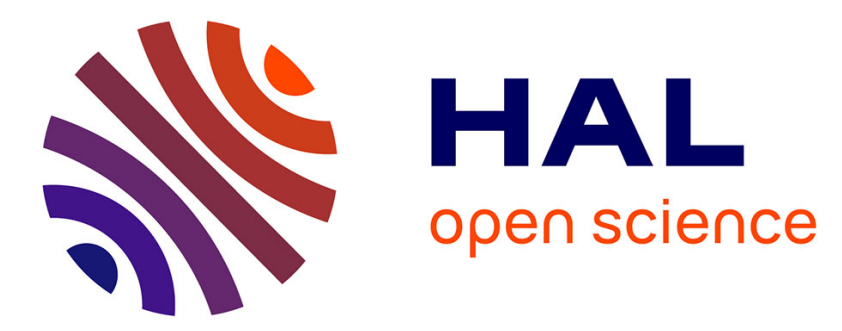

\title{
Mechatronic design of the Gyrolift verticalization wheelchair
}

Lambert Trenoras, Uréné Gregory, Eric Monacelli, Vincent Hugel

\section{To cite this version:}

Lambert Trenoras, Uréné Gregory, Eric Monacelli, Vincent Hugel. Mechatronic design of the Gyrolift verticalization wheelchair. IEEE/ASME International Conference on Advanced Intelligent Mechatronics (AIM2014), 2014, Besançon, France. pp.1308-1313, 10.1109/AIM.2014.6878263 . hal-01723804

\section{HAL Id: hal-01723804 \\ https://hal-univ-tln.archives-ouvertes.fr/hal-01723804}

Submitted on 13 Feb 2020

HAL is a multi-disciplinary open access archive for the deposit and dissemination of scientific research documents, whether they are published or not. The documents may come from teaching and research institutions in France or abroad, or from public or private research centers.
L'archive ouverte pluridisciplinaire HAL, est destinée au dépôt et à la diffusion de documents scientifiques de niveau recherche, publiés ou non, émanant des établissements d'enseignement et de recherche français ou étrangers, des laboratoires publics ou privés.

\section{(c)(1)}

Distributed under a Creative Commons Attribution| 4.0 International License 


\title{
Mechatronic Design of the Gyrolift Verticalization Wheelchair
}

\author{
Lambert Trenoras $^{1}$, Unéné Gregory ${ }^{2}$, Eric Monacelli $^{3} \&$ Vincent Hugel $^{4}$
}

\begin{abstract}
This paper presents the mechatronic design of the Gyrolift chair, a new type of wheelchair associated with a personal transporter and equipped with a verticalization system. The verticalization system is designed to help users to reach a standing posture. This module allows disabled people to move from the seated position to the standing position. Thanks to this system people can stand safely and interact with objects in the environment that cannot be reached from the seated postion. The major contribution of this research is the design of a mechatronic system, defined by the morphology of the user, to enable suitable verticalization. A biomechanical model is also detailed to define morphological trajectories for the embedded verticalisation system. Experiments were carried out to evaluate and validate the system.
\end{abstract}

\section{INTRODUCTION}

This work aims at improving the mobility of disabled people or people subject to a loss of mobility. Some robotic devices were designed to relieve the life of disabled people, by increasing their autonomy [1] or by helping them to stand from a seated position [2]. Commercial products already exist like the powered wheelchairs iBot [3] or the Genny [4]. Exoskeletons like the Mina [5], or the HAL [6], were proposed for paraplegic people to help them to get verticalised and to use their lower limbs again. However these systems remain too cumbersome and difficult to use. Therefore $69.8 \%$ paraplegics suffering from spinal-cord injuries prefer to use a manual wheelchair to move around [7]. But people with serious mobility trouble cannot use manual wheelchairs and could benefit from assisting systems adapted to their needs.

The lack of motion capabilities has a negative impact on the body such as physiological and biochemical disturbances [8], [9]. The advantage to move from a seated to a standing posture is twofold. First, the user can immediately feel a physiological relief when verticalization starts. As a matter of fact, the standing position improves blood circulation, intestinal transit and bones consolidation for people who usually remain seated or lying down. Second, verticalised users can have access to objects out of reach from the seated posture like cupboards and shelves. Standing users can also feel a certain level of comfort in the practice of daily activities such as washing, cooking, etc.. Furthermore, the wheelchair associated with a verticalisation system presents an actual social interest in matter of communication, allowing

${ }^{1,3,4}$ L.Trenoras, E.Monacelli and V.Hugel are with the Interactive robotics team, LISV - Laboratoire d'Ingénierie des Systemes de Versailles, Université de Versailles Saint-Quentin-en-Yvelines, 10-12 avenue de l'Europe, 78140 Vélizy-Villacoublay, France

${ }^{2}$ U.Gregory is with the Department of Electrical Engineering/French South African Institute of Technology, Tshwane University of Technology, Pretoria, South Africa lambert.trenorasdisv.uvsq. fr

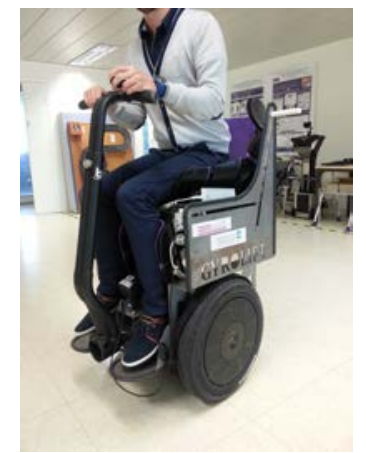

(a) Seated Position

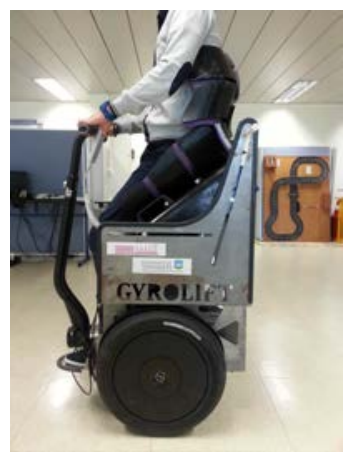

(b) Verticalised Position
Fig. 1: Gyrolift Wheelchair : Verticalization module upon personal transporter

the user to talk to other people while standing. The communication between a seated person and a standing person can create a psychological issue insofar as the seated person can unconsciously has a feeling of inferiority, let alone cervical issues.

This work presents a specific mechatronic device, named Gyrolift. The mobile part of the Gyrolift is a personal transporter. A new verticalisation approach was introduced to equip the mobile part with a verticalization system to help the user to stand comfortably (Fig. 1). Unlike the existing lift elevating devices incorporated into wheelchairs, the Gyrolift allows paraplegic people to move continuously, even when standing. The verticalisation motion requires accurate control in order to limit disturbances on the system.

This first Gyrolift prototype is dedicated to paraplegic users who suffer from low spinal cord injury, but can still control their arms, thorax and head. The Gyrolift was sized according to an entire anthropomorphic body model. This sizing was also used to find a well suited trajectory for the user's verticalisation that prevents his center of mass from moving horizontally, therefore keeping the personal transporter base motionless. Studies on the Hanavan model [10], and medicine and biomechanical studies carried out by Braune and Fischer [11], Dempster [12] or Seward [13] tried to improve the modelling of the human body to obtain realistic limb masses.

The present study aims to validate the mechatronic design, together with the center of mass trajectory, while preserving comfort and stability. The comfort concept is defined from all the feelings sensed by each user who practised the Gyrolift and the verticalisation.

The next section describes the robotic device and its characteristics. The third section is devoted to the theoretical 

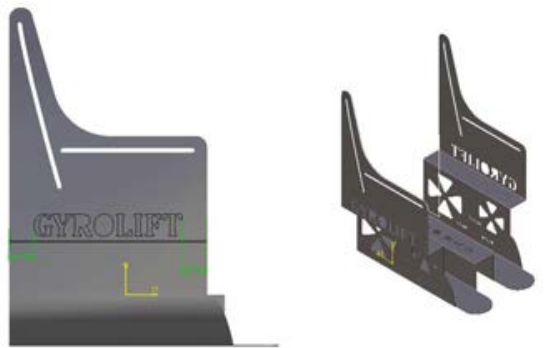

(a) Laser-cut guides

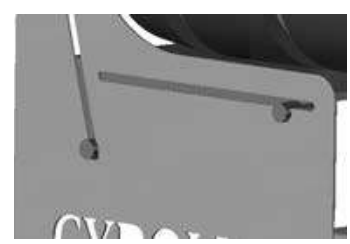

(b) Spacers

Fig. 2: Laser-cut guides onto the steel plate and spacers

model that defines the verticalisation. The fourth section presents the experiments carried out with the Gyrolift. The fifth section discusses the results obtained in this study.

\section{ROBOTIC DEVICE}

The Gyrolift is different from other lift elevating wheelchairs, since it allows the users to move in the verticalised position, benefiting from the displacement capabilities of the personal transporter.

The Gyrolift system is composed of:

- a personal mobile transporter,

- a verticalization system,

- a complete seat with guides for the thighs and the tibias,

- laser-cut steel frames.

The autonomy of the transporter is $40[\mathrm{~km}]$.

The chair and the verticalisation system were designed using a Computer-Aided Design software (CAD). The system weighs $40[\mathrm{~kg}]$ for a technically embedded weight of $120[\mathrm{~kg}]$. Spacers are placed on the laser-cut steel frames to define the trajectory of the seat. They are fixed onto a PVC U-shaped plate (Fig.2b).

\section{A. The seat}

The seat, seat-back and leg rest (Fig. 3a) were being sized according a biomechanical model (see section III) to design a custom-made product that can be manufactured thanks to a $3 \mathrm{D}$ printer. The different parts are then wrapped into a thermoformed foam (Fig. 3b).

The shape of these three parts was designed with the help of an occupational therapist to ensure the holding and comfort of the dorsal, lumbar regions and thighs and legs. Actually, a suited design for the seat limits bedsores and helps consolidation of bones. These parts are mounted onto a PVC plate.

When the user is seated, legs are constrained; ties attach the lower pelvis, thighs, and tibia to the chair. This version does not include rest arms; we assume that hands are

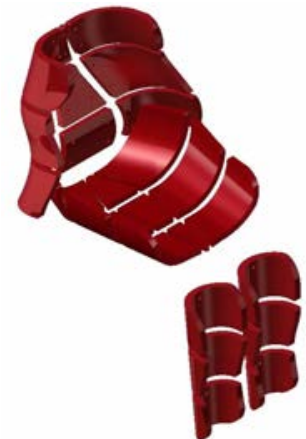

(a) Seat-Back, Seat and Leg rest

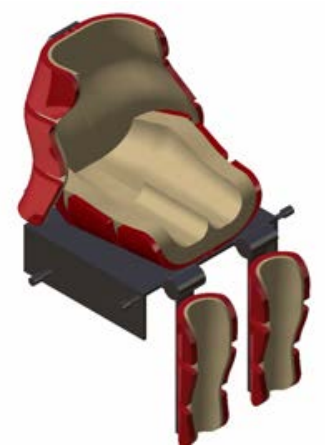

(b) Seat with thermoformed foam
Fig. 3: Seat

grasping the front handlebar. This front handlebar can be removed. Handles located on each part of the seat are here to help the transfer.

\section{B. Verticalisation device}

A linear actuator drives the verticalisation. It has a nominal force of $1500[N]$. The associated battery pack can deliver an electric charge of $2.9[A h]$ at the rated voltage of $24 \mathrm{~V} \mathrm{DC}$. The actuator is fixed to the steel U-shaped plate on one side and on the PVC U-shaped plate on the other side (Fig. 4). These two fixations are passive degrees of freedom. The user pilots the linear actuator thanks to an open-loop controller. The linear actuator moves the seat from a seated to a standing position along a guided trajectory thanks to the spacers inside the lateral frames.

The verticalisation device is composed of mechanical guide frames, laser-cut onto the steel plate (Fig.2a) that are fixed on the personal transporter footplate. These guides provide the trajectories for the knees and the hips. The guided trajectory for the knees' movements is a straight line. For the hips movement, guided trajectories are defined by an ellipse curve. This ellipse trajectory allows a stable verticalisation.

\section{Definition of the trajectory}

The trajectories allow users to be verticalised properly on the personal transporter base. The verticalisation control system should not disturb the system of the chair and needs to be accurate. The personal transporter uses the feedback of
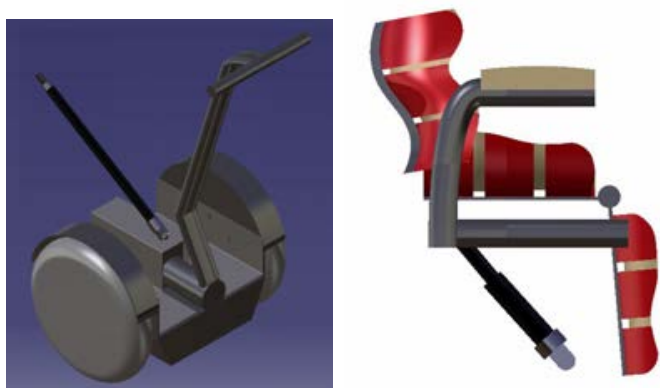

Fig. 4: Linear actuator fixation 


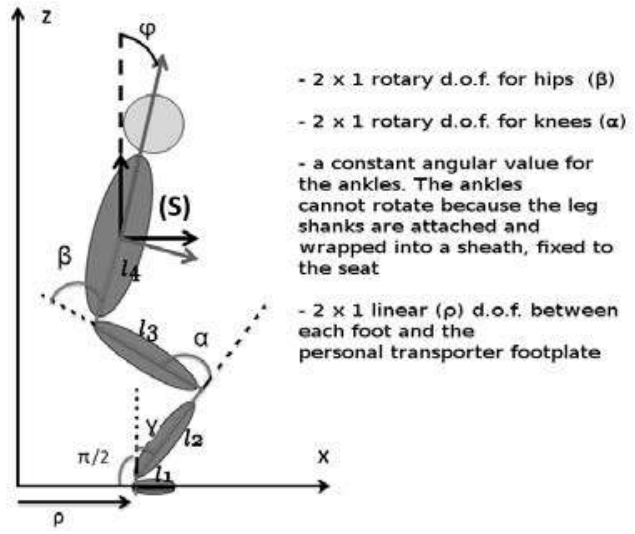

Fig. 5: Kinematic model for the lower limbs. $\varphi$ and $\gamma$ are desired parameters.

the body center of mass. If the user leans forward/backward, the personal transporter moves forward/backward. The user must be stabilized to stand up in a comfortable way.

\section{BIOMECHANICAL MODEL}

The biomechanical model was first used to analyse some verticalisation trajectories on simulation. The purpose was to define a suited trajectory and to visualize the effects of various trajectories on the user.

The simulation model is inspired by the digital humanoid models defined by Gravez et al. [14]. The definition method allows to automatically create a virtual model of a humanoid biped with proper distribution of each body part according to the user's mass and the height.

\section{A. Kinematic modelling of inferior limbs}

Since our objective consists of verticalising people with mobility troubles, the kinematic model of Gravez et al. was simplified. We defined 3 active degrees of freedom, distributed as depicted on Fig. 5. Arms' movements were not taken into account because the hands were assumed to grasp the front handlebar, therefore having low dynamic impact on the model. The angle of the ankles was set constant, equal to $\pi / 2$. This value was fixed according to the usual value of classical lift elevating wheelchairs.

\section{B. Mass distribution}

The model is decomposed into ten segments (Fig. 6). In

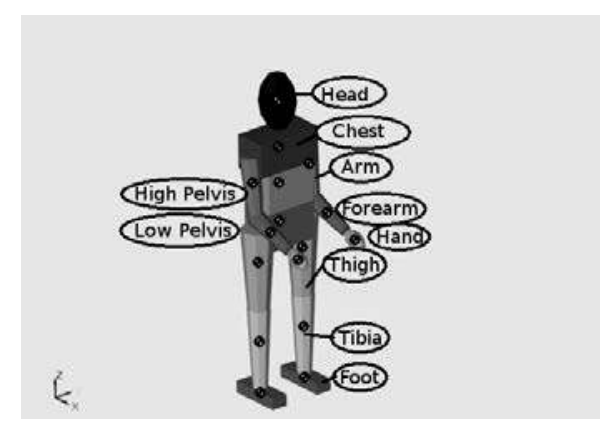

Fig. 6: Model this model, the mass $m_{i}$ of the body segment $i$ is given thanks to the following linear expression, where $M$ is the mass of the subject :

$$
m_{i}=a_{i} M+b_{i} \quad \text { for } \quad i=1, \ldots, n
$$

The positions $h g_{i}$ of each limb's center of mass influences the dynamics of the system. As proposed by Gravez et al., we used a constant value $d_{i}$, that is a ratio of the length of the limb from the proximal point, to express $h g_{i}$ :

$$
h g_{i}=d_{i} H \quad \text { for } \quad i=1, \ldots, n
$$

\section{Limbs dimensions}

Limbs dimensions are expressed as a function of the height of the subject, namely $H$ :

$$
h_{i}=p_{i} H \quad \text { for } \quad i=1, \ldots, n
$$

$h_{i}$ is the length of the main segment composing body part $i$. As for the masses, we want to get the limbs sizes thanks to a single parameter, namely the height of the model.

Similarly we defined parameters of volumes and inertia matrices. Body parts were defined as parallelepipeds for the lower pelvis, upper pelvis and chest, as truncated cones for the arms, forearms, thighs and tibias, and as ellipsoids for the head and the hands.

\section{Inverse kinematics}

We reused this biomechanical model to get verticalisation trajectories and study their effects on the variation of the center of mass. We defined a trajectory for the virtual endeffector $S$ (Fig. 5), to generate the desired standing position. The inverse kinematic of our model is defined as:

$$
\left\{\begin{array}{l}
\rho=l_{2} \sin \gamma+l_{3} \cos \left(-2 \gamma+\arcsin \left(\frac{l_{1}+l_{2} \cos \gamma+l_{4} \sin \gamma-z}{l_{3}}\right)\right) \\
+l_{4} \cos \varphi+x \\
\alpha=\gamma-\arcsin \left(\frac{l_{1}+l_{2} \cos \gamma+l_{4} \sin \gamma-z}{l_{3}}\right) ; \\
\beta=-\frac{\pi}{2}-2 \gamma+\arcsin \left(\frac{l_{1}+l_{2} \cos \gamma+l_{4} \sin \gamma-z}{l_{3}}\right)+\varphi
\end{array}\right.
$$

where $l_{1}, l_{2}, l_{3}$, and $l_{4}$ respectively designate the foot height, tibia length, thigh length, and the length between the hip and the end-effector.

The control model is defined with an open-loop inverse kinematic model $Q=f(S)$ [Eq. 4], that allows defining joint angles, velocity and acceleration.

\section{E. Optimal trajectory}

Using the biomechanical model we focused on different trajectories of verticalisation. The objective was to determine a verticalisation trajectory that prevents the forward motion of the center of mass that typically occurs during a classical verticalisation (Fig. 7a).

In a first series of tests, we applied a linear vertical trajectory to the end-effector $(S)$, which is the center of the trunk:

$$
\left\{\begin{array}{l}
X(t)=X_{\text {init }} \\
Z(t)=Z_{\text {init }}+a
\end{array}\right.
$$




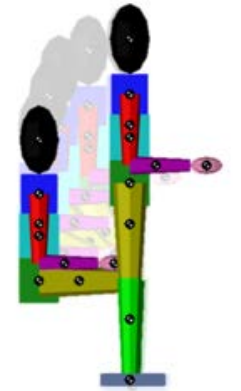

(a) Classical Verticalisation

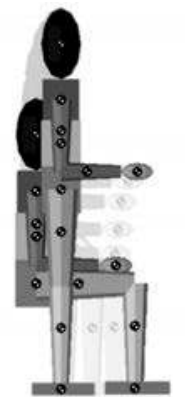

(b) New verticalisation
Fig. 7: Verticalisation

where

- $a$ is the length of the thigh, $a=L_{\text {Thigh }}$.

- $X_{\text {init }}$ and $Z_{\text {init }}$ are the initial coordinates of the endeffector on the $X$ and $Z$ axes.

Fig. 8 shows that the center of mass moves backward along an ellipsoid trajectory. Therefore the linear movement leads to an undesired backward disturbance.

Thanks to these tests, we decided to apply an opposite ellipsoid trajectory for $(S)$ to prevent the disturbing backward motion. The resulting ellipsoid shape is defined as follows:

$$
\left\{\begin{array}{l}
X(t)=X_{i n i t}-b \cos (\alpha(t)) \\
Z(t)=Z_{i n i t}+a \cos (\alpha(t))
\end{array}\right.
$$

Where

- $b$ is the frontal displacement of the center of mass during the verticalisation,

$b=X_{\text {CoMseat }}-X_{\text {CoMstand }}$.

$X_{\text {CoMseat }}$ is the position on the $\mathrm{X}$ axis of the center of mass when the model is in the seated position.

$X_{C o M}$ stand is the position on the $\mathrm{X}$ axis of the center of mass when the model is in the standing position.

$X_{\text {init }}$ and $Z_{\text {init }}$ are the initial coordinates of the endeffector on the $X$ and $Z$ axes.

Fig. 9a shows the trajectory of the end-effector for different morphologies. We can notice the impact of the

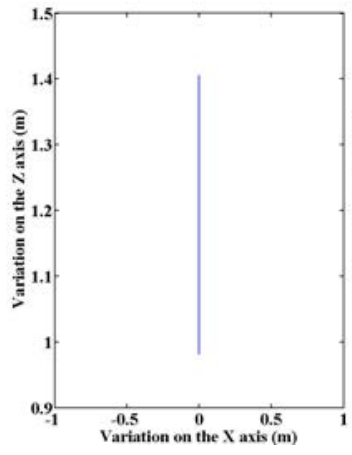

(a) End-Effector Trajectory

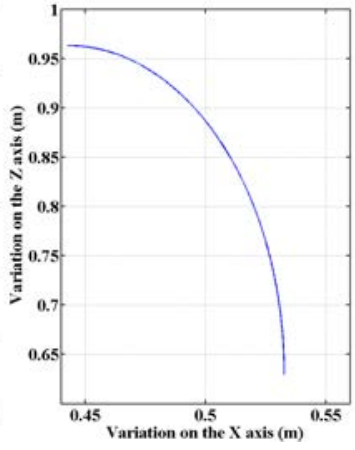

(b) CoM Trajectory

Fig. 8: End-Effector and CoM trajectories

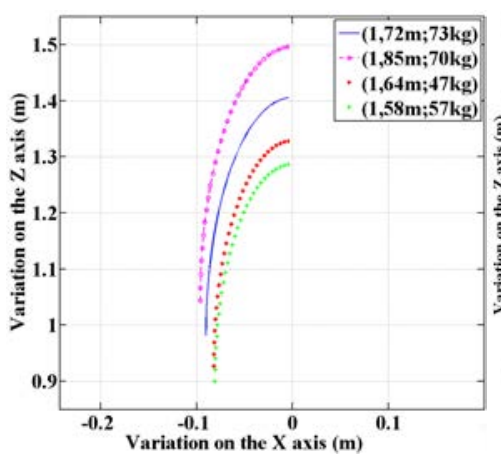

(a) End-effector trajectories for different people $(\mathrm{m})$

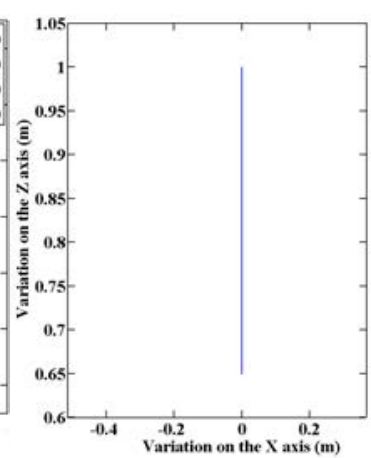

(b) CoM trajectory $(\mathrm{m})$
Fig. 9: Trajectories in the sagittal plane

height/weight couple on the trajectory. But the trajectory of the center of mass remains vertical (Fig. 9b).

Thanks to the ellipsoid shape, we come up with a new type of verticalisation as illustrated on Fig. 7b. We can notice that the user can move slightly backward. This is allowed thanks to the $\rho$ parameter. Variations of $\alpha$ and $\beta$ are used to achieve the trajectory of the end-effector and keep accuracy of the desired parameters.

Compared with the classical verticalisation (Fig. 7a), the new verticalisation proposed (Fig. 7b \& Fig. 9a) is more stable since it prevents the user's center of mass from moving horizontally, therefore avoiding undesired forward motion of the transporter base.

\section{EXPERIMENTAL STUDY}

The morphology of a particular user was taken as a reference to design the wheelchair. We calculated the anthropomorphic model of this user using the parameterized biomechanical model described previously, and we obtained the optimal trajectory for his verticalisation, e.g. specific trajectories of the knees and the hips for each guide.

A set of experiments was carried out with the verticalisation wheelchair for validation. Users had to master this wheelchair model that is completely different from traditional ones.

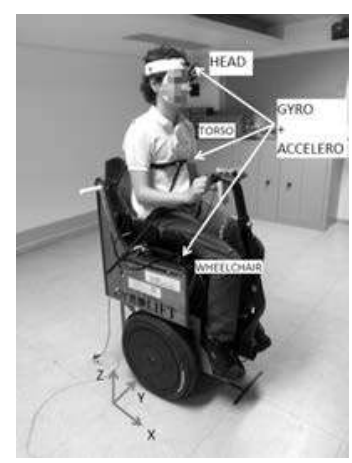

(a) Accelero/Gyro Sensors

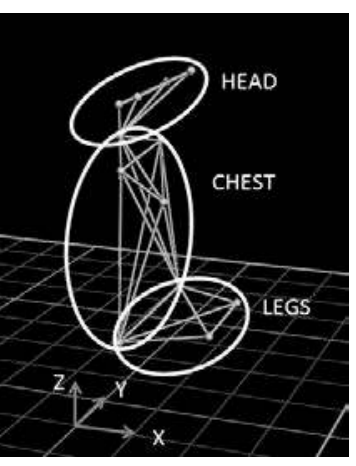

(b) Vicon configuration
Fig. 10: Sensor configuration and axis definition 
The objective was to get the user verticalised without any forward or backward motion of the system. First, we analysed the compensation movement executed by different users during their verticalisation on the Gyrolift. Second, we examined the trajectory of their center of mass.

\section{A. Motion capture}

In the first stage, we used a Vicon system to capture body movements (Fig. 10b). The Vicon system was composed of six optical cameras that gave grayscale information, from which we could get the centres of the markers up to submillimetre accuracy. We put markers on the user's head, neck, torso and thighs, and defined three bodies, head, chest and legs linked respectively by a spherical joint and a revolute joint.

In the next stage, we set up a system based on three pairs of accelerometer/gyro sensors (Fig. 10a). According to previous studies devoted to human body motion capture [15], the suited fusion of data coming from accelerometer and gyroscope sensors proved to be efficient. A pair of sensors was fixed to the seat to track the movement of the seat/thighs. A second pair was attached to the user's torso. The third pair was placed on the user's head.

\section{B. Protocol}

These experiments serve as pre-tests before conducting actual tests with paraplegic people the Gyrolift was designed for. The objective was to validate the accuracy of the mechatronic system and the trajectory parameterization.

We selected a panel of twelve people: three groups of four persons. In the first group the persons were nearly as tall as the initial user whose size and morphology were used to design the Gyrolift. This group included two experts in ergonomy, and an occupational therapist to get their feedback on the system. The second group consisted of taller users (height greater than $10[\mathrm{~cm}]$ ). Shorter users belonged to the third group (height less than $10[\mathrm{~cm}]$ ).

We gave each user a $15[\mathrm{~min}]$ time to get accustomed to the wheelchair. Then we placed markers and sensors on them. Once a user was ready to be verticalised, he could trigger the verticalisation motion, which started the recording process of the accelerometer/gyroscope sensors data. Each user was asked to execute 5 verticalisation motions.

\section{RESULTS}

First, we used the Vicon motion-capture system to obtain the position of the chest and the compensation - if it exists - made by the user to control the verticalization wheelchair. Since the personal transporter is controlled by the position of the center of mass, we call compensation the chest movement of the user to correct a possible displacement of the personal transporter. We analysed the compensation due to the morphology differences and the impact on the trajectory.

Second, we used inertial data of the medium group to analyse the defined trajectory on an individual. We extracted linear accelerations along the $x$ (front/rear) and $z$ (up/down)

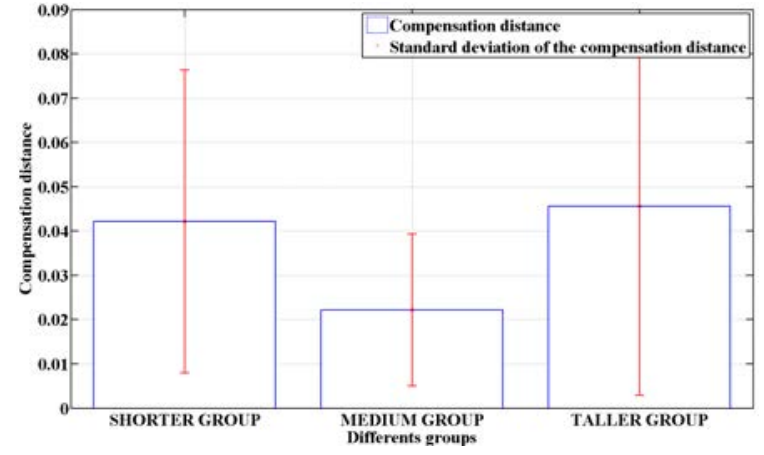

Fig. 11: Body compensation distance [m] for each users' group.

axes, and the angular velocity along the $y$ axis (pitch) to get angles of the thighs, the chest and the head in the sagittal plane. These angles were then reincorporated into the biomechanical model. We run the simulation again using the biomechanical model in order to obtain the position of the virtual end-effector and the position of the center of mass during the verticalisation.

\section{A. Vicon Motion Capture}

Thanks to the Vicon motion-capture system, we analysed the effect of user movements on the personal transporter, and the variation of the users's compensation during the verticalisation. The user's compensation is defined by the difference between the trajectory of his hips and the trajectory of his chest.

Results are depicted on Fig. 11. We notice that groups compensate the movement of the personal transporter due to the error created by their verticalisation. We can also note that people who do not belong to the medium group have to compensate more. This compensation ranges between $2[\mathrm{~cm}]$ and $5[\mathrm{~cm}]$. This variation can be considered as limited, and cannot be seen as an overcompensation. However, we observe that a user who executes the verticalisation with his own trajectory compensates two times less than a user who executes the verticalisation with an unsuitable trajectory.

\section{B. Inertial Data and Theoretical model}

In a second phase, we used angles and angular velocities as inputs to the biomechanical model to recreate the verticalisation. We compared the measured trajectories of the actual end-effector obtained by the model with the optimal trajectories relative to the user calculated by Eq. (6). We also observed the trajectory of the user's center of mass, and compared it with the optimal trajectory. We proceeded this way for all five verticalisations of each user.

Fig. 12 shows the trajectory for a trial run of a user.

Since trajectories and errors of the end-effector and the center of mass are linked, we calculated the average of the Root Mean Square Error (RMSE) for the end-effector trajectory, and for the COM trajectory.

Table I gives the RMSE values of the user's end-effector and COM relative to the medium group. The RMSE values 


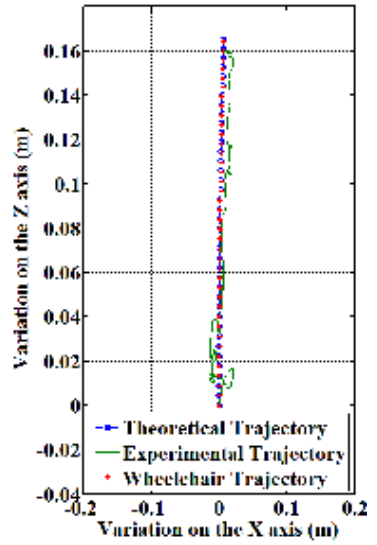

(a) End-Effector Trajectories

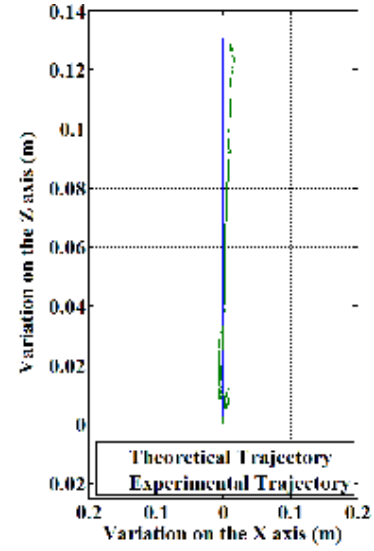

(b) CoM Trajectories
Fig. 12: End-Effector and CoM trajectories

TABLE I: Root Mean Square Error

\begin{tabular}{|c||c|c|}
\hline- & $\begin{array}{c}\text { RMSE of } \\
\text { the End-Effector }(\mathrm{m})\end{array}$ & $\begin{array}{c}\text { RMSE of } \\
\text { the Center of Mass }(\mathrm{m})\end{array}$ \\
\hline Medium Group & $1.93 \mathrm{E}-2$ & $1.02 \mathrm{E}-2$ \\
\hline
\end{tabular}

remain below $2[\mathrm{~cm}]$. A trajectory was considered as suitable when the error was less than $10[\mathrm{~cm}]$.

\section{DISCUSSION}

This research study came up with a new type of verticalization wheelchair that was specifically designed to be used by paraplegic people.

Experiments carried out on a panel of users lead to results that validate the proposed model. The two experts in ergonomics and the occupational therapist reported that they felt comfortable with the Gyrolift, that it was quick to learn and reactive. However, they also mentioned a sensation of little discomfort due to the fact that the seat and the rest legs did not suit them. This feedback will be considered for future releases.

Compensation errors (Fig. 11) confirm the importance of a suited trajectory for the couple height/weight. A suitable trajectory allows reducing by $50 \%$ the error between the designed trajectory and the user's own ideal trajectory. The user has to compensate this error. That is why it is necessary to match the trajectory with the user's morphology as much as possible. We also validated the mechatronic design of the Gyrolift insofar as it allows to perform the verticalization adequately.

In a future release we intend to investigate the possible integration of new parameters that could improve these results. The robotic device can probably give better results thanks to a closed control loop and a higher number of active degrees of freedom. A new and more exoskeletal Gyrolift is under way. Although the biomechanical model used gives satisfactory results, it is only a kinematic model. Dynamic effects could be taken into account. In addition the movements of the arms, and the use of floating masses instead of a rigid body can improve the accuracy of the model.

\section{CONCLUSION}

This paper has presented the research achievements in the framework of the Gyrolift project, which consists of designing and building a mechatronic module dedicated to verticalization, to be fixed onto a wheelchair. A specific mechatronic system was designed, with specific verticalization trajectory, and was embedded into a personal transporter. Experiments were carried out on a population of valid people whose legs were fixed to the chair. Results obtained from this first series of evaluation are promising. Actually this new wheelchair could be adopted by people suffering from lower spinal cord injury. The next step will consist of designing a second version of the Gyrolift wheelchair with an enhanced control system and additional active degrees of freedom. This new system will aim to control the verticalisation of a larger panel of people of different morphology without necessarily changing the system. This system will be designed to target a larger population of disabled people with more severe spinal cord injuries.

\section{REFERENCES}

[1] S. Dubowsky, F. Genot, S. Godding, H. Kozono, A. Skwersky, H. Yu, and L. S. Yu, "Pamm-a robotic aid to the elderly for mobility assistance and monitoring: A helping-hand for the elderly," in Proceedings of the IEEE International Conference on Robotics and Automation, 2000, pp. $570-576$.

[2] D. Chugo, T. Asawa, T. Kitamura, S. Jia, and K. Takase, "A rehabilitation walker with standing and walking assistance," in Proceedings of the IEEE/RSJ International Conference on Intelligent Robots and Systems, 2008, pp. 260-265.

[3] IBot, http://www.ibotnow.com/.

[4] G. Mobility, http://www.gennymobility.com/.

[5] P. D. Neuhaus, J. H. Noorden, T. J. Craig, T. Torres, J. Kirschbaum, and J. E. Pratt, "Design and evaluation of mina: A robotic orthosis for paraplegics," in Proceedings of the IEEE International Conference on Rehabilitation Robotics, 2011, pp. 1-8.

[6] A. Tsukahara, Y. Hasegawa, and Y. Sankai, "Standing-up motion support for paraplegic patient with robot suit hal," in Proceedings of the IEEE International Conference on Rehabilitation Robotics, 2009, pp. 211-217.

[7] M. Berkowitz, Spinal cord injury: An analysis of medical and social costs. Demos Medical Publishing, 1998.

[8] D. Czell, R. Schreier, R. Rupp, S. Eberhard, G. Colombo, and V. Dietz, "Influence of passive leg movements on blood circulation on the tilt table in healthy adults," Journal of neuroengineering and rehabilitation, vol. 1, p. 4, 2004.

[9] V. Dietz, R. Müller, and G. Colombo, "Locomotor activity in spinal man: significance of afferent input from joint and load receptors," Brain, vol. 125, no. 12, pp. 2626-2634, 2002.

[10] E. P. Hanavan Jr, "A mathematical model of the human body," DTIC Document, Tech. Rep., 1964.

[11] W. Braune, O. Fischer, P. Maquet, and R. Furlong, Determination of the moments of inertia of the human body and its limbs. SpringerVerlag, 1988.

[12] W. T. Dempster, "The anthropometry of body action," Annals of the New York Academy of Sciences, vol. 63, no. 4, pp. 559-585, 1955.

[13] D. Seward, A. Bradshaw, and F. Margrave, "The anatomy of a humanoid robot," Robotica, vol. 14, no. 04, pp. 437-443, 1996.

[14] F. Gravez, O. Bruneau, and F. B. Ouezdou, "Analytical and automatic modeling of digital humanoids," International Journal of Humanoid Robotics, vol. 2, pp. 337-359, 2005.

[15] T. Sakaguchi, T. Kanamori, H. Katayose, K. Sato, and S. Inokuchi, "Human motion capture by integrating gyroscopes and accelerometers," in Proceedings of the IEEE/SICE/RSJ International Conference on Multisensor Fusion and Integration for Intelligent Systems, 1996, pp. $470-475$. 